\title{
RENDIMENTO DE FEIJ ÃO E DE GRÃO-DE-BICO EM FUNÇÃO DE FOSFATO SOLÚVEL APLICADO EM COVAS OU NA LINHA DE SEMEADURA ${ }^{(1)}$
}

\author{
M. $\operatorname{VOSS}^{(2)}$, M.S. PARRA ${ }^{(3)} \&$ A. D. CAMPOS $^{(4)}$
}

\begin{abstract}
RESUMO
E ntre os macronutrientes, o fósforo é o que com maior freqüência proporciona aumento na produtividade das culturas. Tem-se demonstrado que a obtenção de nitrogênio por algumas das simbioses rizóbio-legumi nosas exige teores maiores de fósforo do que a planta adubada com nitrogênio. Visando aumentar a eficiência no aproveitamento de fósforo no solo sem incrementar a dose de adubação, testaramse modos de localização de adubo com fosfato solúvel para as culturas de feijão e de grão-de-bico. $O$ feijão, em ensaio instalado em J aguapitã (PR), foi semeado, em outubro de 1986, em um Latossolo Vermelho-E scuro com $113 \mathrm{~g} \mathrm{~kg}^{-1}$ de argila e $2,2 \mathrm{mg} \mathrm{dm}^{-3}$ de P. $O$ adubo fosfatado foi distribuído a $7 \mathrm{~cm}$, e as sementes a $3 \mathrm{~cm}$ de profundidade, ambos no sulco de semeadura. Comparou-se a adubação no sulco com a adubação em covas, em doses de 50 e de $100 \mathrm{~kg} \mathrm{ha}^{-1} \mathrm{de}_{2} \mathrm{O}_{5}$, como superfosfato triplo (SFT). Incluiu-se um tratamento extra com $25 \mathrm{~kg} \mathrm{ha}^{-1} \mathrm{de} \mathrm{P}_{2} \mathrm{O}_{5} \mathrm{em}$ covas, além de testemunha sem adubação fosfatada. $O$ feijão foi semeado em covas. $O$ ensaio com grão-de-bico foi instalado em Londrina (PR ), em junho de 1987, em um Latossolo Roxo com $760 \mathrm{~g} \mathrm{~kg}^{-1}$ de argila e $3,6 \mathrm{mg} \mathrm{dm}^{-3}$ de P. A adubação foi feita a $7 \mathrm{~cm}$ de profundidade, e a semeadura a $3 \mathrm{~cm}$ de profundidade. Nesse ensaio, foram testados, nas parcelas, os tratamentos: testemunha sem rizóbio, inoculação com rizóbio e sem inoculação + $30 \mathrm{~kg} \mathrm{ha}^{-1}$ de $\mathbf{N}$ e, nas subparcelas, a adubação fosfatada em linha ou em covas. $\mathbf{O}$ grão-de-bico foi semeado em linha. Foram avaliados os teores de $\mathbf{N}$, $P, Z n$ e Mn nas folhas de feijão, bem como a produtividade e a nodulação de feijão e de grão-de-bico. Não houve nodulação em feijão e a tendência para maior nodulação em grão-de-bico, quando a adubação foi aplicada em covas, não foi significativa. Houve aumento de cerca de $40 \%$ no rendimento do feijão e de $31 \%$ no de grão-debico pela adubação em covas, em relação à adubação em linha. E m feijão, a eficiência na utilização de $P$ em covas foi de $15,5,10,4$ e $7,4 \mathrm{~kg}$ de grãos por $\mathrm{kg}$ de $\mathrm{P}_{2} \mathrm{O}_{5}$, respectivamente, para 25, 50 e $100 \mathrm{~kg} \mathrm{ha}^{-1}$ de $\mathrm{P}_{2} \mathrm{O}_{5}$. Em contraste, a adubação em linha proporcionou eficiência de utilização próxima a 4. A aplicação de adubo em covas pode ser uma alternativa para aumentar a eficiência da adubação fosfatada em feijão e em grão-de-bico.
\end{abstract}

Termos de indexação: eficiência de utilização de fósforo, localização da adubação, fixação biológica de nitrogênio, nodulação.

\footnotetext{
(1) Trabal ho parcialmente apresentado no XXIII Congresso Brasileiro de Ciência do Solo, Porto Alegre, 21 a 27 de julho de 1991 e parcialmentena XVIII Reunión Latinoamericana de Rizobiologia. Santa Cruz de la Sierra. Bolívia, 24 a 28 de setembro de 1996. Recebido para publicação em novembro de 1996 e aprovado em janeiro de 1998.

(2) Engenheiro-Agrônomo, Pesquisador da EMBRAPA-Centro Nacional ePesquisa doTrigo. Caixa Postal 451, CEP 99001-970 Passo Fundo (RS). E-mail:Voss@enpt.embrapa.br.

(3) Engenheiro-Agrônomo, Pesquisador do Instituto Agronômico do Paraná - IAPAR. Caixa Postal 481, CEP $86001-970$ Londrina (PR).

(4) Técnico Agrícola.IAPAR. Caixa Postal 481, CEP 86001-970 Londrina (PR).
} 


\title{
SUMMARY: YIELD OF COMMON BEAN AND CHICKPEA AS A FUNCTION OF SOLUBLE PHOSPHATE APPLIED IN PITS OR IN THE SEEDING ROW
}

\begin{abstract}
Phosphorus is themacronutrient that most often contributes to increasing the productivity of crops in Brazil. It has been shown that certain Rhizobium-plants symbiosis require higher levels of phosphorus, as compared to the nitrogen- fertilized plants. Several different ways of placing soluble phosphate were tested for both common beans and chickpea crops, with the objective of increasing the efficiency of using phosphorus availabl ein thesoil without increasing fertilizer amount. The common bean crop set up in J aguapitã (PR), was sown in October, 1986 in an Oxisol with $113 \mathrm{~g} \mathrm{~kg}^{-1}$ of clay and $2.2 \mathrm{mg} \mathrm{dm}^{-3}$ of $P$. The fertilizer was distributed at $7 \mathrm{~cm}$ depth and theseeds at $3 \mathrm{~cm}$ depth, both being pl aced in therow. Row ferti lizati on was compared to pit fertilizati on using 50 and $100 \mathrm{~kg} \mathrm{ha}^{-1}$ of $\mathrm{P}_{2} \mathrm{O}_{5}$, as triplesuperphosphate I $n$ addition to the control, without phosphatefertilizati on, an extra treatment using $25 \mathrm{~kg} \mathrm{ha}^{-1}$ was al so included. The common bean was seeded in pits. The chickpea trial was set up in Londrina (PR) in J une 1987, in an Oxisol with $760 \mathrm{~g} \mathrm{~kg}^{-1}$ of clay and 3,6 $\mathrm{mg} \mathrm{dm}^{-3}$ of P. The fertilizer was placed at the depth of $7 \mathrm{~cm}$ and the seeds at $3 \mathrm{~cm}$. The treatments Rhizobium-free control, inoculation with Rhizobium, and without inoculation $+30 \mathrm{~kg} \mathrm{~N} . \mathrm{ha}^{-1}$, weretested in thepl ots, whilein thesubpl ots thetests involved phosphatefertilization in rows or in pits. Thechi ckpea was seeded in therow. The contents of N, P, Zn and $\mathrm{Mn}$ in the common bean leaves, as well as the productivity and nodulati on of common bean and chickpea, were assessed. There was no root nodulation in the common beans, and a tendency to greater nodul ati on in thechickpea when using pit fertilizati on was not statistically si gnificant. There was an increase of about $40 \%$ in thecommon bean yield and of $31 \%$ in chickpea as a result of pit fertilization, as compared to row fertilization. The efficiency of using $P$ in pits in the common bean was of $15,5,10.4$ and $7,4 \mathrm{~kg}$ of grain for $\mathrm{kg}$ of $\mathrm{P}_{2} \mathrm{O}_{5}$ for 25,50 , and $100 \mathrm{~kg}$ ha-1 $\mathrm{P}_{2} \mathrm{O}_{5}$, respectively. In contrast, row fertilization showed an index close to 4. Pit application of soluble phosphate may be an alternative choice to help increase the efficiency of phosphorus fertilization.
\end{abstract}

Index terms: efficiency in P utilization, fertilizer placement, biological nitrogen fixation, nodulation.

\section{INTRODUÇÃO}

Os teores de fósforo são originalmente baixos na mai oria dos sol os do Brasil, em virtude de seu el evado poder de imobilização do nutriente adicionado (Raij et al., 1982). Por isso, são exigi das quantidades muito grandes de adubo para el evar o teor de fósforo disponível em todo o volume do solo explorado pelo sistema radicular.

Um contato menor entre o adubo fosfatado e as partículas do solo limita a velocidade e a intensidade de fixação do P. O uso de adubo granulado tem esse objetivo. No Brasil, Sousa \& Volkweiss (1987) quantificaram o gradiente de concentração de fósforo, que se forma radial mente a partir de um grânulo de superfosfato triplo, e encontraram à distância de 5 a $10 \mathrm{~mm}$ teores de até $4.000 \mu \mathrm{g} \mathrm{cm}^{-3}$ de P extraível no solo, 121 dias após sua aplicação.

O conhecimento da proximidade de esgotamento de recursos finitos, como o do fosfato, enfatiza a necessidade de assegurar a maior eficiência possível no seu uso. N esse sentido, resumindo as informações levantadas por um grupo detrabal ho de especialistas brasileiros em adubação fosfatada, Raij et al. (1982), recomendaram, entre outras ações, mais pesquisas sobre a localização de adubos fosfatados. Em dez regiões do Paraná, a adição de $40 \mathrm{~kg}$ ha-1 de $_{2} \mathrm{O}_{5}$ nas linhas, em solos de baixo teor em fósforo disponível, el evou a produtividade do feijão de 650 para cerca de
$1.000 \mathrm{~kg} \mathrm{ha}^{-1}$ (Parra et al., 1980). Baseando em dados deCobra N eto et al. (1971), para atingir esse aumento de $350 \mathrm{~kg}$ de grãos de feijão, a adição de apenas $7,2 \mathrm{~kg} \mathrm{ha}-1$ de $\mathrm{P}_{2} \mathrm{O}_{5}$ bastaria, caso todo o fósforo adicionado fosseaproveitado pela planta. Por outrolado, no caso de leguminosas, para o bom funcionamento da simbiose, geral mente são requeridos teores de $\mathrm{P}$ mais al tos do que para a planta à qual se forneça nitrogênio mineral (Munns, 1977). O feijão (Graham \& Rosas, 1979) ea ervilha (Voss et al., 1987a) são exemplos disso.

U ma forma de aumentar o fósforo disponível no sol o, para mel horar a fixação biológica e o rendimento de grãos sem, entretanto, el evar desnecessariamente as doses de recomendação de $P$, poderia ser a de maior concentração da adubação, tal como se consegue com a aplicação em covas. Esse modo de adubação é praticado por pequenos produtores, que usam semeadeira-adubadeira manual (saraquá ou matraca), ou mesmo enxada. Del inearam-se, então, dois ensaios, um com feijão e outro com grão-de-bico, com o objetivo de comparar a aplicação de SFT em covas com a feita em linha contínua no sulco de semeadura.

\section{MATERIAL E MÉTODOS}

E nsaio com feijão - I nstal ou-se o ensaio em 10/09/ 1987, no município deJ aguapitã, ao norte do Estado do Paraná, em um Latossol oVermel ho-E scuro, sem cultivo 
de feijão há pelo menos 10 anos, com as seguintes características químicas etexturais: $\mathrm{pH}\left(\mathrm{CaCl}_{2}\right): 4,6$; $\mathrm{Al}$ : $1,9 \mathrm{mmol}_{\mathrm{c}} \mathrm{dm}^{-3}, \mathrm{H}+\mathrm{Al}: 44,2 \mathrm{mmol}_{\mathrm{c}} \mathrm{dm}^{-3}, \mathrm{Ca}$ : $17,5 \mathrm{mmol}_{\mathrm{c}} \mathrm{dm}^{-3}, \mathrm{Mg}: 13,2 \mathrm{mmol}_{\mathrm{c}} \mathrm{dm}^{-3}, \mathrm{~K}: 1,0 \mathrm{mmol}_{\mathrm{C}} \mathrm{dm}^{-3}$; C: $10 \mathrm{~g} \mathrm{~kg}^{-1}$; P: 2,2 mg dm-3; areia: $860 \mathrm{~g} \mathrm{~kg}^{-1}$; argila: $130 \mathrm{~g} \mathrm{~kg}^{-1}$; silte: $10 \mathrm{~g} \mathrm{~kg}^{-1}$.

Utilizou-se o delineamento deblocos ao acaso, com 4 repetições. Os tratamentos foram: 1 ) Testemunha sem fósforo, 2) $50 \mathrm{~kg} \mathrm{ha}^{-1} \mathrm{de}_{2} \mathrm{O}_{5}$ em linha, 3) $100 \mathrm{~kg} \mathrm{ha}^{-1} \mathrm{de}$ $\mathrm{P}_{2} \mathrm{O}_{5}$ em linha, 4) $50 \mathrm{~kg} \mathrm{ha}^{-1}$ de $\mathrm{P}_{2} \mathrm{O}_{5}$ em covas, entre covas de semeadura, 5) $100 \mathrm{~kg} \mathrm{ha}^{-1}$ de $\mathrm{P}_{2} \mathrm{O}_{5}$ em covas, entrecovas de semeadura, 6) $50 \mathrm{~kg}$ ha-1 $\mathrm{deP}_{2} \mathrm{O}_{5}$, sob as covas desemeadura, 7) $100 \mathrm{~kg} \mathrm{ha}^{-1} \mathrm{de}_{2} \mathrm{O}_{5}$, sob as covas de plantio, 8) $25 \mathrm{~kg}$ ha-1 $\mathrm{deP}_{2} \mathrm{O}_{5}$, sob as covas de plantio. O fertilizanteusado foi oSFT. As parcelas constituíramse de quatro linhas de quatro metros de comprimento, espaçamento entre linhas de 0,5 metro.

O preparo do sol o foi feito com uma aração seguida de duas gradagens. A adubação fosfatada foi feita à profundidade de cerca de $7 \mathrm{~cm}$. Empregou-se SFT granulado, peneirado, com vistas em obter grânulos com mai or uniformidade, com diâmetro próximo de $4 \mathrm{~mm}$. Como planta indicadora, utilizou-se o cultivar de feijão IAPAR 16. Nos tratamentos de adubação em covas, utilizou-se implemento manual (saraquá) com um bico mais fino do que o comum, para colocar oSFT em covas.

A semeadura foi feita também com saraquá, em covas separadas cerca de $20 \mathrm{~cm}$, à profundidade aproximada de $3 \mathrm{~cm}$. E m al guns tratamentos (4 e 5), - SFT foi colocado a cerca de $10 \mathrm{~cm}$ de cada cova de semeadura, ficando, portanto, no meio do intervalo das covas. $\mathrm{E} m$ outros tratamentos de adubação em covas $(6,7,8)$, o SFT foi colocado abaixo da cova de semeadura. Para a aplicação do SFT em linha, abriuse um sulco no solo, empregando-se uma enxadinha, até a profundidade de cerca de $7 \mathrm{~cm}$, fazendo-se a adubação manual mente. Fez-se, então, a cobertura do adubo no sulco, com cerca de $4 \mathrm{~cm}$ de solo, e as sementes foram colocadas, em grupos de 4, em covas distanciadas $20 \mathrm{~cm}$ umas das outras. Portanto, em todos os tratamentos, a semeadura foi feita em covas.

O potássio foi colocado com matraca, a cerca de $5 \mathrm{~cm}$ ao lado da cova de sementes, à razão de $30 \mathrm{~kg}$ ha-1 de $\mathrm{K}_{2} \mathrm{O}$ sob a forma de cloreto de potássio. Não se adubou com nitrogênio. Utilizou-seinoculanteturfoso à razão de $800 \mathrm{~g}$ ha-1, aderido às sementes com açúcar diluído em água a $20 \mathrm{~g} \mathrm{~L}-1$. Doze dias após a emergência, procedeu-se ao desbaste, deixando-se 25 plantas por $\mathrm{m}^{2}$. Ocorreu ataque inicial de vaquinha (Diabrotica speciosa), controlado com aplicação de inseticida de contato. As ervas daninhas foram capinadas um dia antes da semeadura e três semanas após a emergência do feijoeiro.

Por ocasião do florescimento, fez-se avaliação da nodulação, amostrando-se as raízes dentro das dimensões de 0,2 $\mathrm{m}$ de largura, de 0,2 $\mathrm{m}$ de profundidade e de um metro de comprimento ao longo da linha desemeadura. As fol has das plantas retiradas para a avaliação anterior foram utilizadas para análise de nitrogênio, fósforo, zinco e manganês. Tanto as análises de tecido como as de solo foram feitas conforme Muzilli et al. (1978). Aos 89 dias do plantio, procedeu-se à colheita das duas linhas centrais do feijoeiro, correspondendo à área de $3,2 \mathrm{~m}^{2}$ por parcela.

Ensaio com grão-de-bico - I nstal ou-se o ensaio em 02/06/87, em Londrina, no norte do Estado do Paraná, em um Latossol o Roxo, com a seguinte análise química e textural: $\mathrm{pH}\left(\mathrm{CaCl}_{2}\right): 5,1 ; \mathrm{Al}: 0,0 \mathrm{mmol}_{c} \mathrm{dm}^{-3}$ Ca: $48,2 \mathrm{mmol}_{\mathrm{c}} \mathrm{dm}^{-3}$; $\mathrm{Mg}: 16,8 \mathrm{mmol}_{\mathrm{c}} \mathrm{dm}^{-3}, \mathrm{~K}$ : 9,7 mmol $\mathrm{dm}^{-3}$; $\mathrm{C}: 15 \mathrm{~g} \mathrm{~kg}^{-1}$ e P: $3,6 \mathrm{mg} \mathrm{dm}^{-3}$, areia: $100 \mathrm{~g} \mathrm{~kg}^{-1}$; argila: $760 \mathrm{~g} \mathrm{~kg}^{-1}$; silte: $140 \mathrm{~g} \mathrm{~kg}^{-1}$.

Empregou-se o delineamento de blocos ao acaso, em esquema de parcelas subdivididas, com quatro repetições. Nas parcelas, foram testados os tratamentos: testemunha sem inoculação, inoculação com rizóbio, sem inoculação $+30 \mathrm{~kg} \mathrm{ha}^{-1}$ de $\mathrm{N}$ e, nas subparcelas, os tratamentos: adubação PK em covas e em linha. $\mathrm{O}$ adubo, com fórmula $\left(\mathrm{N}-\mathrm{P}_{2} \mathrm{O}_{5}-\mathrm{K}_{2} \mathrm{O}\right)$ 0-30-10, foi aplicado na profundidadede $4 \mathrm{~cm}$ abaixo do nível desemeadura, na dose de 200 kg ha-1, em todos os tratamentos.

A adubação nitrogenada nas parcelas com 30 kg ha-1 deN foi feita com sulfato de amônio, em cobertura, 25 dias após a emergência. Semeou-se o cultivar de grãode-bico (Cicer arietinum) C 81491, em subparcelas de 2,4 4 m, em quatrolinhas espaçadas de 0,6 m entresi.

A semeadura foi feita em sulco deaproximadamente $3 \mathrm{~cm}$ de profundidade, manual mente, em todos os tratamentos, col ocando-se uma semente a cada $5 \mathrm{~cm}$. A pós desbaste, a densidade média foi de 24,7 plantas por $\mathrm{m}^{2}$. Houve necessidade de replantio, em julho de 1987. A cultura desenvolveu-se num período muito seco.

A nodulação foi avaliada durante a floraçãoformação de vagens, 70 dias após a emergência. Foram col hidas, em novembro de 1987, as duas linhas centrais na extensão de $3 \mathrm{~m}$, para determinar o peso de grãos. Para a análise estatística do número de nódulos, fezse a transformação dos dados para $\sqrt{\mathrm{X}+1}$.

\section{RESULTADOS E DISCUSSÃO}

No ensaio com feijão, a nodulação foi nula e, conseqüentemente, não houve contribuição do nitrogênio atmosférico para ofeijão, via simbiose. Por essa razão, conclui-se quetodo o nitrogênio obtido pela planta adveio das reservas do solo. A produtividade obtida deve ter sido um pouco restringida por deficiência de nitrogênio. No quadro 1, observa-se que o teor de nitrogênio mostrou-se inferior nos tratamentos com maior produção de grãos, refletindo maior diluição pelo maior crescimento das plantas. No mesmo quadro, verifica-se que os teores de $\mathrm{P}$ e de Zn não mostraram al terações significativas nos vários tratamentos estudados no período de floração, o que foi inesperado, uma vez que os tratamentos envolveram doses de $\mathrm{P}$. Os teores de $\mathrm{Mn}$ atingiram valores mai ores, quando se adicionou mais $\mathrm{P}$ em covas. A acidez provocada pela dissolução do SFT poderia ser a causa desse aumento da absorção de $\mathrm{Mn}$, (Malavol ta, 1981). Não se procedeu à anal ise de outros elementos no tecido da planta. 
Os dados deprodutividadedegrãos estãoapresentados no quadro 2. Embora os valores tenham sido baixos, verificou-se efeito de tratamentos.

Houve resposta a doses e modos de adubação fosfatada. Por outro lado, dentro da mesma dose, as duas modal idades de adubação em covas não diferiram entresi. Dentro de cada modo de adubação, a produção aumentou segundo a dose de fosfato. Com a adubação em linha, o aumento foi de 51 e $96 \%$, respectivamente, para 50 e para $100 \mathrm{~kg} \mathrm{ha}^{-1} \mathrm{de}_{2} \mathrm{O}_{5}$, quando comparado ao tratamento-testemunha sem P. J á a adubação em covas proporcionou acréscimos de 121 e 173\%, respectivamente, para as doses de 50 e de 100 kg ha-1 de $\mathrm{P}_{2} \mathrm{O}_{5}$ em relação à testemunha. Assim, dentro de cada dose, o aumento proporcionado pela localização em covas foi cerca de $40 \%$ maior do que o obtido com adubação em linhas, seja com 50 ou com 100 kg de $\mathrm{P}_{2} \mathrm{O}_{5}$. A comparação dos dois modos de adubação fica mais contrastante, quando se verifica quea produção proporcionada por $25 \mathrm{~kg}$ ha-1 de $\mathrm{P}_{2} \mathrm{O}_{5}$ em covas eqüivaleu à de $100 \mathrm{~kg}$ ha-1 de $\mathrm{P}_{2} \mathrm{O}_{5}$ em linha.

No único trabalho analisado sobre comparação de adubação em covas e em linha, no sulco de semeadura, de Miranda (1970), não foram obtidas diferenças na produtividade de feijão, cv. Rico 23, em sol os argilosos de Viçosa (MG). O autor aplicou $400 \mathrm{~kg} \mathrm{ha-1}$ de superfosfato simples e $60 \mathrm{~kg} \mathrm{ha}^{-1}$ de $\mathrm{K}_{2} \mathrm{O}$ por ocasião do plantio.

Analisando a eficiência de utilização da adubação fosfatada no presente ensaio, por meio da relação do aumento da produção de feijão obtido por unidade de $\mathrm{P}_{2} \mathrm{O}_{5}$ aplicada, observou-se que os índices para o fósforo aplicado em linha foram próximos a 4, semelhantes aos calculados por Raij et al. (1982), para ensaios realizados no Sudeste do Brasil, enquanto, na adubação em covas, os índices foram superiores, passando a 7,4, 10,4 e 15,5 para 100, 50 e 25 kg ha-1 de $\mathrm{P}_{2} \mathrm{O}_{5}$, respectivamente.

Quadro 1. Teores de fósforo, nitrogênio, manganês e zinco das folhas do feijão cv. IAPAR 16 por ocasião do florescimento, em função de doses e modos de adubação com fosfato solúvel

\begin{tabular}{|c|c|c|c|c|c|}
\hline \multirow{2}{*}{$\begin{array}{c}\text { Tratamento } \\
\left(\mathbf{P}_{2} \mathbf{O}_{5}\right)\end{array}$} & \multirow{2}{*}{ Localização } & \multicolumn{4}{|c|}{ Teor de nutriente } \\
\hline & & $\mathbf{P}$ & $\mathbf{N}$ & Mn & Zn \\
\hline $\mathrm{kg} \mathrm{ha}^{-1}$ & & 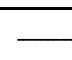 & - & $-\mathrm{ms}$ & - \\
\hline 0 & & 2,2 & 46 & $153 d^{(1)}$ & 36 \\
\hline 50 & em linha & 2,3 & 42 & $185 \mathrm{~cd}$ & 33 \\
\hline 100 & em linha & 2,6 & 40 & $214 \mathrm{bcd}$ & 31 \\
\hline 25 & em cova, na cova de semeadura & 2,2 & 42 & $229 \mathrm{bcd}$ & 30 \\
\hline 50 & em cova, entre covas de semeadura & 2,4 & 38 & $280 a b$ & 33 \\
\hline 50 & em cova, na cova de semeadura & 2,3 & 40 & 208 bcd & 30 \\
\hline 100 & em cova, entre covas de semeadura & 2,2 & 39 & 357 a & 30 \\
\hline 100 & em cova, na cova de semeadura & 2,3 & 37 & $329 a b$ & 30 \\
\hline
\end{tabular}

(1) Letras iguais indicam igualdade estatística pelo teste de Duncan ao nível de $5 \%$.

Quadro 2. Rendimento absoluto e relativo do feijão IAPAR 16, e eficiência na utilização de fosfato (E UF ) em função de níveis e modos de adubação com superfosfato triplo em um Latossolo Vermelho-E scuro

\begin{tabular}{|c|c|c|c|c|}
\hline \multirow{2}{*}{$\begin{array}{c}\begin{array}{c}\text { Tratamento } \\
\left(\mathbf{P}_{\mathbf{2}} \mathbf{O}_{\mathbf{5}}\right)\end{array} \\
\mathrm{kg} \mathrm{ha}^{-1}\end{array}$} & \multirow[t]{2}{*}{ Localização } & \multicolumn{2}{|c|}{ Rendimento de grãos } & \multirow[t]{2}{*}{$E U F^{(2)}$} \\
\hline & & $\mathrm{kg} \mathrm{ha}^{-1}$ & $\%$ & \\
\hline 0 & & $432 c(1)$ & 100 & \\
\hline 50 & em linha & $656 \mathrm{bc}$ & 151 & 4,48 \\
\hline 100 & em linha & $847 a b$ & 196 & 4,15 \\
\hline 25 & em cova, na cova de semeadura & $821 a b$ & 190 & 15,50 \\
\hline 50 & em cova, entre covas de semeadura & $959 a b$ & 222 & 10,54 \\
\hline 50 & em cova, na cova de semeadura & $949 a b$ & 220 & 10,34 \\
\hline 100 & em cova, entre covas de semeadura & $1.186 \mathrm{a}$ & 274 & 7,54 \\
\hline 100 & em cova, na cova de semeadura & $1.175 \mathrm{a}$ & 272 & 7,43 \\
\hline
\end{tabular}

(1) Letras iguais indicam igualdade pelo teste de Duncan a 5\%. ${ }^{(2)}$ EUF- eficiência na utilização de fosfato: $\mathrm{kg}$ de grãos por kg de $\mathrm{P}_{2} \mathrm{O}_{5}$. 
Os resultados relevantes do ensaio com grão-debico são apresentados no quadro 3. A adubação em covas produziu aumento de $31 \%$ no rendimento de grãos em relação à adubação em linha. A nodulação somente ocorreu nos tratamentos inoculados. O peso e o número de nódul os no modo de adubação em covas não apresentaram diferenças estatísticas, em rel ação à nodulação do grão-de-bico com aplicação de P em sulco.

A semelhança no rendimento de grãos entre os tratamentos $\operatorname{com} N$, ou com inoculação ou sem $N$ nem inoculação, dentro de cada modo de adubação (dados não mostrados), deve-se, provavelmente, ao período muito seco ocorrido durante o ensaio. No caso dos tratamentos inoculados, embora as estirpes usadas tenham tido sua eficiência confirmada para o mesmo tipo de solo por Voss et al. (1987b), encontrou-se, no presente ensaio, um terço ou menos da nodulação obtida no ensai o dos autores citados. Os tratamentos: inoculação, nitrogênio e sem inoculação não afetaram o rendimento de grãos, razão por que foram agrupados dentro dos tratamentos de modos de adubação para a análise estatística.

A tendência de maior nodulação no grão-de-bico no modo de aplicação de $\mathrm{P}$ em covas do que em sulcos (significativo apenas a $10 \%$ de probabilidade) merece ser investigada em condições climáticas mais favoráveis.

Não foi encontradonenhum trabal ho, na literatura, sobre a localização de adubo em covas em grão-debico.

Os efeitos dos tratamentos de ambos os ensaios podem ser atribuídos à aplicação do fósforo, uma vez queo potássio foi aplicadonas mesmas doses e deigual modo para o feijão e, além do mais, no caso do grãode-bico, o teor de potássio no solo era muito el evado. A maior taxa de absorção de $\mathrm{P}$ por parte das raízes em contato com esse nutriente em covas e maior desenvolvimento das raízes próximo à região fertilizada devem explicar a maior eficiência de utilização do $P$ com a localização do adubo em covas. Essas duas alterações foram demonstradas com milho por J ager (1978), Anghinoni \& Barber (1980) e
Klepker \& Anghinoni (1995). Esses autores observaram incremento na taxa da absorção de $\mathrm{P}$ e na densidade de raízes na parte inserida na região mais rica em $P$, em vasos com separação de solo adubado e não adubado. Com isso, ocorreu uma compensação parcial pela menor absorção de $\mathrm{P}$ pelas outras raízes. I nfelizmente, nestes ensai os, não foram determinados esses aspectos.

Mesmo que, em outras condições, as diferenças na eficiência da utilização de $P$ em função dos métodos de aplicação não sejam tão grandes como os obtidos no presente trabalho, é importante considerar o emprego da adubação fosfatada em covas em condições de agricultura de subsistência, em que baixos níveis de produtividade são justificáveis pela limitação do fator capital. No caso do feijão, típica lavoura de subsistência no Brasil, o val or dessas considerações é ressaltado pelo grau elevado de risco que envolve o seu cultivo. Atual mente, o grão-de-bico éuma cultura quetem pouca representatividadeno Brasil, mas pode constituir uma alternativa de renda para o pequeno agricultor.

\section{CONCLUSÃO}

Em solos de baixa disponibilidade de fósforo, a produtividade de feijão e de grão-de-bico em um ano de cultivo indica que, quando a aplicação de fosfato solúvel for feita em covas, é possível diminuir a dose de $\mathrm{P}$ em relação à recomendada para aplicação em linha contínua nos sulcos de semeadura.

\section{AGRADE CIMENTOS}

Ao engenheiro-agrônomo Antonio Veranio, da E mater-PR, à química Olga Bagatini ea Maurício Rosa de Oliveira, pela ajuda na realização deste trabal ho. Aos revisores da Revista Brasileira de Ciência doSolo, pelas excel entes sugestões oferecidas.

Quadro 3. Efeito do modo de adubação de P e K sobre o rendimento e nodulação de grão-de-bico, em um Latossolo Roxo

\begin{tabular}{cccc}
\hline Tratamento $^{(1)}$ & Rendimento de grãos $^{(1)}$ & Peso de nódulos $^{(2)}$ & Nódulos $^{(2)}$ \\
\hline & $\mathrm{kg} \mathrm{ha}^{-1}$ & mg planta $^{-1}$ & número/planta \\
Adubação em covas & $1.518 \mathrm{a}$ & 25,9 & 44,00 \\
Adubação em linhas & $1.156 \mathrm{~b}$ & 14,1 & 13,75 \\
\hline
\end{tabular}

${ }^{(1)}$ Média de 12 repetições. Comparação de médias por Tukey ao nível de $1 \% .{ }^{(2)}$ Média de quatro repetições. Só foram comparados os dois tratamentos inoculados. F (5\%). 


\section{LITERATURA CITADA}

ANGHINONI, I. \& BARBER, S.A Phosphorus influx and growth characteristics of corn roots as influenced by phosphorus supply. Agron. J., 72:685-688, 1980.

COBRA NETO, A.; ACCORSI, W.R. \& MALAVOLTA, E. Estudos sobre a nutrição mineral do feijoeiro (Phaseol us vulgaris L.) var. Roxinho. Anais da ESALQ, 28:257-74, 1971.

GRAHAM, P.H. \& ROSAS, J.C. Phosphorus fertilization and symbiotic nitrogen fixation in common bean (Phaseolus vulgaris L.). Agron. J ., 71:925-927, 1979.

J AGER, A. Localized stimulation of root growth and phosphate uptake in Zea mays L. resulting from restricted phosphate supply. In: HARLEY, J .L. \& SCOTT RUSSEL, R. eds. The soil root interface. New York, Academic Press, 1978. p.391-403.

KLEPKER, D. \& ANGHINONI, I. Crescimento radicular e aéreo do milho em vasos em função do nível de fósforo no solo e da localização do adubo fosfatado. R. Bras. Ci. Solo, 19:403-408, 1995.

MALAVOLTA, E. Manual de química agrícola - adubos eadubações. São Paulo, Ceres, 1981. 596p.

MIRANDA, A.R. Efeito do modo de aplicação dos adubos, no solo, sobre as culturas de amendoim, ervilha e feijão. Viçosa, Universidade Federal deViçosa, 1970. 43p. (Tese de Mestrado)
MUNNS, D.N. Mineral nutrition and the legume symbiosis. In: HARDY, R.W.F \& GIBSON, A.H. eds. A treatise on dinitrogen fixation. Section IV. N ew York, J ohn Wiley \& Sons, 1977. p.353392.

MUZILLI, O.; LANTMANN, A.F.; PALHANO,J .B.; OLIVEIRA, E.L.; PARRA, M.S.; COSTA, A.; CHAVES, J.C.D. \& ZOCOLER, D.C. Análise de sol os: Interpretação e recomendação de calagem eadubação para o Estado do Paraná. Londrina, IAPAR, 1978. 49p. (Circular, 9)

PARRA, M.S.; HOEPFNER, M.A. \& VOSS, M. Adubação do feijão no Estado do Paraná. In: CULTURA DO FEIJ ÃO NO ESTADO DO PARANÁ. Londrina, IAPAR, 1980. p.33-45. (Circular, 18)

RAIJ , B. van; ROSAND, P.C. \& LOBATO, E. Adubação fosfatada no Brasil - apreciação geral, conclusões e recomendações. In: OLIVEIRA, A.J .; LOURENÇO, S. \& GOEDERT, W.J . eds. Adubação fosfatada no Brasil. Brasília, EMBRAPA, 1982. p.928. (Documentos, 21)

SOUSA, D.M. \& VOLKSWEISS, S.J . Reações do superfosfato triplo em grânulos com solos. R. Bras. Ci. Solo, 11:133-140, 1987.

VOSS, M.; CALEGARI, A. \& RIBEIRO, P.G.F. Resposta de ervilha à inoculação com rizóbio e à adubação com fósforo. Londrina, IAPAR, 1987a. 8p. (Informe de Pesquisa, 73)

VOSS, M.; CALEGARI, A. \& RIBEIRO, P.G.F. Resposta de grão de bico à inoculação com rizóbio sob dois níveis de calagem. Londrina, IAPAR, 1987b. 7p. (Informe de Pesquisa, 74) 